\title{
From the library
}

\section{Remembrance of things past}

"Fanon has no criticisms to make of waking dream therapy. One word indicates that he was also interested in going beyond the parameters of the psychotherapy in which he had been trained. He writes: 'Then increasingly small circles appear and scotomize the Negroes.' Scotomize is a very rare word. It has a very peculiar history in French psychoanalysis. It derives from the Latin scotoma and originally meant an obscuration of the part of the field of vision due to a lesion on the retina." (David Macey. Frantz Fanon: a biography. New York: Picador, 2000:138)

\section{Early exposure to pets reduces atopy} related diseases

A population based cohort study of 2531 children in Oslo who were followed from birth to the age of 4 years indicates that early life exposure to pets reduces the risk of developing atopy related diseases such as asthma, eczema, and allergic rhinitis. It is unclear how early life exposure to pets reduces this risk. (Allergy 2001;56:307-12)

\section{Cigarette smoking increases risk of hip} fracture in women

A population based case-controlled study from Sweden investigated post-menopausal women who sustained a hip fracture after minor trauma. Compared with those who had never smoked, current smokers have an increased risk of hip fracture. Duration of smoking, particularly post-menopausal smoking, was more important than the number of cigarettes smoked. Alcohol consumption was not a risk factor for hip fracture and even had a weak inverse association with risk. (Archives of Internal Medicine 2001;61:983-8)

\section{Blocking HIV transmission with} cyclodextrins

Researchers know that cholesterol is essential in the ability of HIV to penetrate cells. Recent studies suggest that by removing cholesterol from cells HIV transmission to these cells can be prevented. Researchers at Johns Hopkins Medical Center have demonstrated that when exposed to the HIV virus cells from which cholesterol had been removed by the use of cyclodextrins resisted infection. These findings may lead to the development of topical creams that might be used to reduce HIV transmission during sexual intercourse. ( $(7 M A$ 2001;86:909)

\section{New approaches to infectious diseases}

New approaches are being developed to combat infectious diseases, which do not use the classic strategies of antibiotic drugs. These new strategies prevent the pathogen from entering and hiding in host cells, thus making it visible to the immune system and allowing the body's own defence systems to eliminate it. Drug resistance, which is the problem in antibiotic therapy, is therefore less likely. Treatments involving this strategy might include use of small molecules to block the surface structure, which pathogens use to recognise and attach to target cells, thus preventing the pathogens from penetrating the cell. (American Scientist 2001;89:406-13)
Smoking increases the risk of renal transplant failure

A cohort study of adult renal allograft recipients was performed at the University of Michigan. This study demonstrates that pretransplant smoking was significantly associated with reduced overall graft survival Cessation of smoking before renal transplantation has beneficial effects on graft survival (Transplantation 2001;71:1752-7)

\section{Interferon treatment of multiple} sclerosis

A groundbreaking study was published last year indicating that interferon beta may reduce the severity and exacerbations of multiple sclerosis. Now, a second randomised prospective trial has been reported from Italy. In this study the use of interferon beta-1a at an early stage of multiple sclerosis had significan positive effects on both the clinical and MR outcomes. No study has yet addressed the question of whether similar results will be documented in patients presenting with optic neuritis. (Lancet 2001;357:1576-82)

The relation of human juvenile haemangiomas and the placenta Juvenile haemangiomas are common benign tumours that characteristically present in the perinatal period with often a rapid growth during the first year of life and in most case subsequent involution. They may cause am blyopia when they involve the periorbital tissues. In a recent study from Arkansas Children's Hospital, a distinct constellation of tissue specific markers was found to be co-expressed by haemangiomas and placental microvessels. These findings suggest a unique relation between haemangiomas and the placenta and may provide data for new hypotheses concerning the origin of these tumours. (Archives of Dermatology 2001; 137:559-70)

Cost effectiveness of vitamin therapy to lower plasma homocysteine levels

More than 10 large prospective studies have been published demonstrating a statistically significant association of basal homocysteine levels and coronary heat disease. Since January 1998, the US Food and Drug Administration has required that all enriched grain products contain $140 \mu \mathrm{g}$ of folic acid per $100 \mathrm{~g}$. Now a cost effective study from the University of California in San Francisco, using the coronary heart disease policy model, has demonstrated that grain fortification with folic acid is predicted to decrease coronary heart disease events by $8 \%$ in women and $13 \%$ in men with comparable reductions in mortality. However, the model projected that, compared with grain fortification alone, treating all patients with known coronary heart disease with folic acid and cyanocobalamin over a 10 year period would result in 310000 deaths and lower costs. Folic acid and cyanocobalamin supplementation would appear to be cost effective in reducing coronary heart disease. ( $(A M A$ 2001;286:936-43)

\section{Animal models developed for the} hantaviruses

The hantavirus family causes several disorders throughout the world. In Europe and Asia, a group of different hantaviruses causes tens of thousands of cases yearly of a disease called haemorrhagic fever with renal syndrome. In the United States, the SIN Nombre virus causes hantavirus pulmonary syndrome (HPS) with a mortality of one third to one half. A recent study by US Army virologists has finally established an animal model to study hantaviruses. This will provide an opportunity to study vaccines and drugs to combat the various forms of hantaviruses. (Science 2001;293:1414-15)

\section{Exposure to soy based formula in} infancy

Soy isoflavins are naturally occurring compounds known to bind to oestrogen receptors in adults, and to act as either oestrogens or anti-oestrogens. Infants fed soy formula receive relatively high doses of phyto-oestrogens during the developmental stage in which permanent changes might theoretically occur. A retrospective study from the University of Iowa has examined adults who as infants participated in a controlled feeding study. The results suggest that exposure to soy formula does not appear to lead to different general health or reproductive outcomes from exposure to cow's milk formula. However, women who had been fed soy formula reported slightly longer duration of menstrual bleeding as well as greater discomfort with menstruation. (FAMA 2001;286:807-14)

\section{Unhappy doctors}

In a recent issue of the $B M F$, Richard Smith outlined the possible reasons why physicians seem to be so dissatisfied with their current situation. While many letters to the editor were written on this topic only a few voices challenged the premise that physicians were dissatisfied with their profession. Now a study from Massachusetts shows that physician job satisfaction has fallen dramatically in the past 15 years. Less than two thirds of primary care physicians in Massachusetts reported to be satisfied with most professional areas. Even fewer, less than half were content with the amount of time spent with patients, the amount of leisure time they had, and the incentives they received to provide high quality care. Insurance issues were the primary cause of most physicians' dissatisfaction. (Fournal of General Internal Medicine 2001;16:451-9)

\section{Artificial lung, a real possibility}

Researchers at a bioengineering firm in Massachusetts have developed a temporary artificial lung, which is inserted through a vein or leg and guided into the vena cava. The several feet long intravenous membrane oxygenator consists of about 1000 hollow fibre membranes. The catheter can provide about 50\% of adult respiratory needs. Clinical trials are set to begin in Europe in the next year to evaluate this device. ( $(\mathcal{A} A M A 2001 ; 286: 781)$ 